\title{
DESIREE DEMO - a web-based software ecosystem for the personalized, collaborative and multidisciplinary management of primary breast cancer
}

\author{
Nekane Larburu ${ }^{1,2}$, Naiara Muro ${ }^{1,2,3}$, Iván Macía ${ }^{1,2}$ \\ ${ }^{1}$ eHealth and Biomedical Applications, Vicomtech, Donostia-San Sebastian, Spain \\ ${ }^{2}$ Biodonostia, Donostia-San Sebastian, Spain \\ ${ }^{3}$ Sorbonne Universités, UPMC Univ Paris 06, INSERM, Université Paris 13, Sorbonne, Paris Cité, UMR S 1142, LIMICS, \\ Paris, France \\ nlarburu@vicomtech.org
}

\begin{abstract}
Breast cancer is the most overspread cancer in women worldwide, with around 1.7 million new cases every year. Multidisciplinary clinical teams or committees, usually known as Breast Units (BU), are heterogeneous teams composed by all clinical specialist involved in the care of a breast cancer patient (e.g. oncologist, surgeon, ...) that aim to discuss these complex clinical cases from all points of view and in the shortest time to provide best health care. BUs base their decisions on the Clinical Practice Guidelines (CPGs), documents that summarize the latest and best evidence-based medicine. Nevertheless, these documents have some knowledge lacks that make them insufficient when working with complex cases "out of the rule", that may represent the $10-20 \%$ of the cases. To cope with this pitfall, DESIREE proposes a unified ecosystem that manages all the relevant information of the patients and provides support to clinicians when making a clinical decision in a personalized, collaborative and multidisciplinary way. It is composed by three main components: (i) an image-based breast and tumor characterization tool, (ii) a predictive model after breast conservative therapy and radio-biological model, and (iii) three different clinical decision support systems (i.e. guideline-based, experience-based and patient similarity based) that coexist and complement each other to give most personalized and best evidence-based recommendations to the BUs. All these are supported by DESIMS (i.e. DESiree Information Management System), a Security and Access Control module and an image system for image and models visualization.
\end{abstract}

Keywords-breast cancer, decision support system

\section{PROJECT DESCRIPTION}

Breast Units (BU) have limited time to study, analyze and provide best evidence-based healthcare to breast cancer patients. Accomplishing this task successfully requires large knowledge on the last updated Clinical Practice Guidelines (CPGs) and previously studied cases and their outcomes, being a very tedious, complex and stressful task that must be done in a very short time and considering all clinical information of the analyzed patient. The BUs have periodic regular meetings where the clinical cases are discussed among all specialists and a decision is taken by consensus to be applied to the patient. Nevertheless, decisions on previous cases are not thoroughly studied as there is no unified system that integrates all heterogeneous information sources and provides analysis tools for supporting the clinician. Some systems provide purely CPG-based decision support, that rely on latest evidence but give generic recommendations or are not able of coping with particular clinical cases that are complex or there is not sufficient evidence reported yet on the CPGs to cope with them. Hence, these systems may become inefficient and not effective at all when supporting clinicians in the decision-making process. In this context, the web-based software ecosystem DESIREE has been developed for a better coordination and information management during the BU. It is composed by several interoperable modules that rely on a Digital Breast Cancer Patient Model built using standard terminologies and codifications (i.e. NCI Thesaurus, UMLS, SNOMED CT) and formalized using semantic web technologies (i.e. ontologies). This model is capable of defining all relevant information characterizing a breast cancer patient to be used in the decision-making process along with the experience generated in this process. This experience is used later on for going beyond the state of the art of the reported knowledge in the model based on previous cases and outcomes, improving and boosting the process of knowledge discovery.

In the demo, the three main modules that compose DESIREE will be presented:

(i) an image-based breast and tumour characterization tool, which extracts valuable information for diagnosis, treatment, prognosis and follow up from the heterogenous set of image data [1]

(ii) a predictive model after breast conservative therapy and radio-biological model, which provides useful results to the clinician and patient from the very first step before conservative therapy [2]

(iii) three different clinical decision support systems that coexist and complement each other to give most personalized and best evidence-based recommendations to the BUs [3]:

- a guideline based CDSS, which implements various international (e.g. NCCN) and local guidelines

- a similarity based CDSS, that gives insights about previously treated similar clinical cases

- an experience based CDSS, which analyzes all decisions and generates new knowledge from them, augmenting the guidelines to cope with complex cases. 


\section{DESIREE PARTNERS}

The partners involved in the project are the following: Arivis AG, Assistance Publique - Hopitaux de Paris, Bilbomática SA, Exploración Radiológica Especiales S.L., Fundación Onkologikoa Fundazioa, Medical Innovation and Technology Single Member PC, Sistemas Genómicos SL, Houston Methodist and Unviersity of Ulster.

\section{ACKNOWLEDGMENT}

This project has received funding from the European Union's Horizon 2020 research and innovation programme under grant agreement No 690238.

\section{REFERENCES}

[1] Gracia Ocaña M., López-Linares Román, K.,Maclair G., Azpíroz Puente M., Romero Martín J.A., Belloch Ugarte V., Santabárbara J., González Ballester M., Macía I. Breast tissue segmentation and density quantification from MRI using convolutional neural networks. In proceedings Int J CARS (2018) 13(Suppl 1): 1, s12-s14

[2] R. Salmon, T. C. Nguyen, A.-C. Lesage, B. L. Bass, and M. Garbey, "Clinical study and multiscale modeling to predict the esthetic outcome of Breast Conservative Therapy."

[3] N. Larburu et al., "Augmenting Guideline-based CDSS with Experts' Knowledge," presented at the 10th International Conference on Health Informatics, 2017, pp. 370-376. 